\title{
Salivary alpha-synuclein (total and oligomeric form): potential biomarkers in Parkinson's disease
}

\author{
Hala Shaheen, Sayed Sobhy, Sherine El Mously º, Marwa Abuomira and Mohamed Mansour
}

\begin{abstract}
Background: Parkinson's disease (PD) is one of the most common degenerative diseases of the central nervous system (CNS). Alpha-synuclein (A-syn) plays a critical role in the pathogenesis of PD. The close relation between the salivary glands and the CNS could render the A-syn secretions in the saliva useful biomarkers for PD.

Aim of the work: To study the salivary A-syn levels in a cohort of PD Egyptian patients and to correlate these Asyn levels with the patients' clinical data and disease severity.

Patients and methods: Twenty-five PD patients and 15 age- and sex-matched healthy subjects, as a control group, were enrolled. Evaluation of PD patients was performed using the Unified Parkinson's Disease Rating Scale (UPDRS) and modified Hoehn and Yahr scale (HYS). Samples of the saliva were analyzed using the enzyme-linked immunosorbent assay (ELISA) technique for the specific anti A-syn total and anti A-syn oligomer (A-synolig).

Results: There was a statistically significant increase in A-synolig level and A-synolig/A-syn total ratio and a decrease in A-syn total level among PD patients. A statistically significant increase in A-synolig level was detected among patients having bradykinesia and rigidity as predominant symptoms. Also, there was a statistically significant positive correlation between A-synolig level and the disease duration. No statistically significant correlation was found between A-syn concentrations and disease severity.
\end{abstract}

Conclusion: Salivary A-syn total and A-synolig can be used as potential biomarkers for PD diagnosis.

Keywords: Parkinson's disease, Biomarkers, Salivary alpha-synuclein

\section{Introduction}

Parkinson's disease (PD) is known to be the second most common neurodegenerative disease. It affects $1-2 \%$ of the population over the age of 60 [1]. As the clinical diagnosis is challenging, misdiagnosis is common to occur. Thus, there is a need for disease-specific biomarkers for early diagnosis and adequate tracking of $\mathrm{PD}$ progression aiming to improve the patients' outcome. Given its critical role in PD pathogenesis, alphasynuclein (A-syn) may be a useful biomarker [2]. In physiological conditions, A-syn is expressed in a monomeric form (A-synmon) [3]. In PD, A-synmon aggregates into A-syn oligomers (A-synolig) which in turn convert into mature amyloid fibrils, leading to the

* Correspondence: smm02@fayoum.edu.eg

Faculty of Medicine, Fayoum University, Keman Fares area, Fayoum City 63611, Egypt

(c) The Author(s). 2020 Open Access This article is distributed under the terms of the Creative Commons Attribution 4.0 International License (http://creativecommons.org/licenses/by/4.0/), which permits unrestricted use, distribution, and reproduction in any medium, provided you give appropriate credit to the original author(s) and the source, provide a link to the Creative Commons license, and indicate if changes were made. formation of Lewy bodies (LB) and Lewy neuritis [4]. Soluble A-synolig are present in larger amounts in the brain of PD patients than in those of healthy subjects and it leads to neuronal cell death, being therefore the main neurotoxic form of A-syn [5]. The submandibular glands are involved in synucleinopathy of PD [6, 7]. Consequently, saliva (typically free of blood contamination) seems to be an ideal biofluid to be studied for Asyn secretions [8]. Salivary A-syn total was lower, while A-synolig and A-synolig/A-syn total ratio were higher in PD patients compared with controls [9]. Other previous studies showed similar results $[8,10]$. Accordingly, we thought to study the salivary A-syn levels in a cohort of PD Egyptian patients also to correlate these A-syn levels with the patients' clinical data and disease severity.

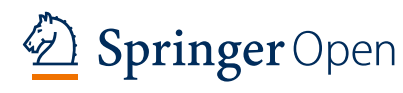




\section{Patients and methods}

This is a case control study that was carried out in the Neurology department, Fayoum University Hospitals, in the period from July 2016 to April 2018. Twenty-five PD patients were enrolled in the study after fulfilling the criteria for diagnosis of idiopathic PD based on the British Brain Bank criteria [11]. Fifteen healthy subjects matched with the patients' age and sex were included as a control group. The former came to the Neurology clinic seeking medical advice for headache or spondylosis.

We excluded participants (whether patients or healthy subjects) having cardiovascular or cerebrovascular diseases, diabetes mellitus, autoimmune diseases, chronic inflammatory diseases, and hematological neoplasms. Also, participants suffering from salivary gland and oral cavity pathologies have been excluded from the study.

All patients and controls were subjected to thorough history taking, general medical, and neurological examination. For the evaluation and staging of PD, we used the Unified Parkinson's Disease Rating Scale (UPDRS) [12]. This scale is subdivided into four parts: part I for mentation, behavior, and mood; part II for activities of daily living; part III for motor examination; and finally, part IV for complications of therapy. For PD staging, we applied the modified Hoehn and Yahr scale (HYS) [13].

PD patients were classified into tremor dominant (TD) phenotype (mean tremor score: mean bradykinesiarigidity score $\geq 1.5$ ) and bradykinesia-rigidity dominant (BRD) phenotype (mean tremor score: mean bradykinesiarigidity score $\leq 1$ ) referring to an older study [14].

The recruited patients were either drug naive or in the on/off stage. The pharmacological state of the patients was assessed and calculated as L-dopa equivalent daily dose (LEDD) for each drug as described in a previous study [15].

Regarding the salivary sample collection, each participant provided $3 \mathrm{ml}$ of saliva to be analyzed using the enzyme-linked immunosorbent assay (ELISA) for A-syn total and A-synolig. The samples were collected, processed, and then stored according to the protocol adopted by Vivacqua and colleagues [9].

Samples were analyzed in the laboratory of the Clinical Pathology department in Fayoum University Hospitals. The protein concentration was determined by spectrometric measurement at $450 \mathrm{~nm}$ in an appropriate microplate reader. This method was applied to draw a standard curve based on the optical density resulting from the presence of A-syn total and A-synolig in the samples; then, we calculated the concentration of the two protein variants in each sample. Each standard curve of optical density matched the corresponding optical density value in the nanogram value of the protein concentration.
We used the anti A-syn ELISA Kit (Biotech E1313Hu) to determine the A-syn total and the anti A-synolig ELISA Kit (MyBioSource, MBS043824) to determine the A-synolig.

One volume of wash solution was diluted with 19 volumes of distilled water. All reagents and samples were brought to room temperature $\left(18-25^{\circ} \mathrm{C}\right)$ for $30 \mathrm{~min}$ before starting the assay procedures. The standard wells, sample wells, and blank/control wells were set; then, a standard of $50 \mu \mathrm{l}$ was added to each standard well. Also, a sample of $50 \mu \mathrm{l}$ was added to each sample well, and a sample diluent of $50 \mu \mathrm{l}$ was added to each blank/control well. All standards, samples, and sample diluent were pooled in duplicate to the plate. Then, streptavidin horse radish peroxidase (HRP) conjugate reagent was added to each well to be incubated for $60 \mathrm{~min}$ at $37^{\circ} \mathrm{C}$. After aspirating the wells, we washed the plates 4 times by applying a wash buffer. After the final wash, the plate is inverted and dried using an absorbent paper. Chromogen solution A $(50 \mu \mathrm{l})$ and chromogen solution B $(50 \mu \mathrm{l})$ were added to each well and gently mixed then incubate for $15 \mathrm{~min}$ at $37^{\circ} \mathrm{C}$. Finally, a stop solution $(50 \mu \mathrm{l})$ was added to each well. We wait till the color in the wells change from blue to yellow then we read the optical density at $450 \mathrm{~nm}$ within $15 \mathrm{~min}$.

\section{Ethical considerations}

The study was reviewed and approved by the Faculty of Medicine Research Ethical Committee, Fayoum University. An informed written consent was signed by all the participants after explaining to them the objectives of the study and all the steps that will be performed. The confidentiality of their information and their right not to participate in the study were ensured.

\section{Statistical analysis}

Data were collected then analyzed by the Statistical Package for the Social Sciences (SPSS) software version 18 in Windows 7. For qualitative data, simple descriptive analysis in the form of numbers and percentages and arithmetic means such as central tendency measurement were applied. Standard deviations (SD) as measure of dispersion were performed for quantitative parametric data. For normality of quantitative, one-sample Kolmogorov-Smirnov test was performed in each study group then inferential statistic tests were chosen selectively. Student's $t$ test was used to compare measures of two independent groups of quantitative data. Nonparametric Mann-Whitney $U$ test was applied for the variables that are not normally distributed. Bivariate (Pearson) correlation test was used to test the association in between the variables. Finally, the receiver operating characteristic (ROC) curve was used to determine the specificity and sensitivity of both A-syn total 
and A-synolig. The cutoff value for significance was considered at $p$ value $\leq 0.05$.

\section{Results}

The study included 25 PD patients. Their age, sex, clinical characteristics, and LEDD are shown in Table 1. The controls were 15 with a mean age $60 \pm 6.7$ years. They were 10 males (66.7\%) and 5 females (33.3\%) with no statistical difference between them and the PD patients $(p>0.5)$.

UPDRS part 1 ranged from 0 to 6 , part 2 ranged from 5 to 33, part 3 ranged from 10to 50, part 4 ranged from 0 to 6 and finally, the total score ranged from 16 to 90 . Modified HYS ranged from 1 to 3 with a mean of $2.08 \pm$ 0.6. Means \pm SD of both scales are presented in Table 2 .

Concerning the laboratory results, the A-syn total level ranged from 29 to $318 \mathrm{ng} / \mathrm{ml}$ in the patient group and ranged from 119 to $333 \mathrm{ng} / \mathrm{ml}$ in the control group. The level of A-synolig ranged from 13.28 to $63.22 \mathrm{ng} / \mathrm{ml}$ in the patient group and ranged from 22.55 to $52.08 \mathrm{ng} / \mathrm{ml}$ in the control group. The remaining laboratory results are shown in Tables 3 and 4 . There was a statistically significant difference $(p<0.05)$ between the study groups.

Furthermore, there was a statistically significant positive correlation between the A-synolig level and disease duration $(r=0.43 ; p=0.03)$. Meanwhile, there was no statistically significant correlation $(p$ value $>0.05)$ between A-syn total, A-synolig, and the ratio between them with the age, sex, LEDD, modified HYS, and UPDRS among cases.

Concerning the sensitivity and specificity of A-synolig in PD diagnosis according to the ROC curve, the total accuracy was $72.4 \%$ with a sensitivity of $76 \%$ and

Table 1 Age, sex, clinical characteristics, and LEDD of PD patients

\begin{tabular}{lll}
\hline Patients $(N=25)$ & \\
\hline Age in years & $51-75$ years & \\
Mean \pm SD & $60.1 \pm 5.6$ & \\
Disease duration & 6 months-10 years & \\
$\quad$ Mean \pm SD & $3.8 \pm 2.7$ & \\
Sex & & $60 \%$ \\
Male $(N, \%)$ & 15 & $40 \%$ \\
Female $(N, \%)$ & 10 & $80 \%$ \\
Phenotypes & \\
TD $(N, \%)$ & 20 & $20 \%$ \\
BRD $(N, \%)$ & 5 & \\
LEDD & $0-1525 \mathrm{mg}$ & \\
Mean \pm SD & $600 \pm 411.2$ &
\end{tabular}

$S D$, standard deviation; $N$, number; $T D$, tremor dominant; $B R D$, bradykinesiarigidity dominant; $L E D D$, L-Dopa equivalent daily dose; $m g$, milligram
Table 2 Means \pm SD of UPDRS and HYS

\begin{tabular}{cc}
\hline & Mean \pm SD \\
\hline UPDRS & \\
Part 1 & $2.56 \pm 1.6$ \\
Part 2 & $13.4 \pm 8.1$ \\
Part 3 & $29.9 \pm 11.1$ \\
Part 4 & $1.24 \pm 1.5$ \\
Total & $47.2 \pm 19.5$ \\
Modified HYS & $2.08 \pm 0.6$ \\
\hline
\end{tabular}

SD, standard deviation; UPDRS, Unified Parkinson Disease Rating Scale; HYS, Hoehn and Yahr scale

specificity of $60 \%$. Positive predictive value (PPV) was $65.5 \%$ and negative predictive value (NPP) was $71.4 \%$ at cutoff of $41.19 \mathrm{ng} / \mathrm{ml}$. Regarding the sensitivity and specificity of A-syn total in PD diagnosis, the total accuracy was $82.3 \%$ with a sensitivity of $80 \%$ and specificity of $86.7 \%$. PPV was $85.7 \%$ and NPP was $81.2 \%$ at cutoff of $182 \mathrm{ng} / \mathrm{ml}$.

\section{Discussion}

In the present study, a statistically significant difference was found between the study groups as regards A-syn total, A-synolig, and the ratio between them with increased A-synolig and A-synolig /A-syn total ratio and decreased A-syn total level among PD patients. These results agreed with several previous studies [8-10]. The results observed in saliva were mirrored in the cerebrospinal fluid (CSF) [9]. It was suggested that the difference in concentrations between A-syn total and Asynolig can be attributed to the oligomerization of free A-synmon in saliva, which leads to the reduction of Asyn total concentration. An elevated A-synolig level was previously detected in the plasma and CSF of PD patients in comparison with controls [16-18]. BengoaVergniory and co-authors [19] suggested that the presence of A-synolig in biological fluids is a more accurate indicator of the disease and measuring the total levels of A-syn hide the difference in oligomer levels. A-synolig levels were monitored as biomarkers for PD and its progression in plasma and CSF in two previous studies [17, 20]. Obtaining a sample of saliva offers multiple advantages over the currently tested biological fluids (blood and CSF) because it is a simple, non-invasive, and painless procedure that does not require expert training [21].

We found also a higher level of A-synolig among BRD phenotype compared with the TD phenotype. This result may be explained by the association of BRD subtype with more dynamic progression and more severe form of the disease which in turn causes more oligomerization of A-syn [22]. This theory is supported by a neuropathologic study on 27 patients with akinetic-rigid PD and 18 
Table 3 Comparisons of A-syn levels in the different study groups

\begin{tabular}{llll}
\hline A-syn & Patients $(N=25)$, mean $\pm S D$ & Controls $(N=15)$, mean $\pm S D$ & $p$ value \\
\hline A-synolig & $47.8 \pm 11.8$ & $39.2 \pm 9.2$ & $0.02^{*}$ \\
A-syn total & $159.4 \pm 61.6$ & $229.9 \pm 64$ & $0.001^{* *}$ \\
A-synolig/A-syn total ratio & $0.35 \pm 0.18$ & $0.19 \pm 0.08$ & $0.003^{* *}$ \\
\hline
\end{tabular}

$N$, number; $S D$, standard deviation; $A$-syn, alpha-synuclein; $A$-synolig, alpha-synuclein oligomer

"Significant; ${ }^{* *}$ Highly significant

with tremor-dominant PD, and the former group had a greater neuronal loss in the substantia nigra pars compacta [23].

Furthermore, there was a statistically significant positive correlation between A-synolig level and disease duration, but with no significant correlation between disease duration and salivary A-syn total, this means that the longer the duration, the more accumulations of the toxic A-synolig occurs. However, Malec-Litwinowicz and colleagues [24] found no correlation between plasma A-syn concentration and duration of the disease. These contradictory results can be attributed to different sample source with the possibility of the contamination of A-syn samples originating from morphologic blood elements [25].

There was no statistically significant correlation between the levels of A-syn total, A-synolig, and the ratio between them with either the score of modified HYS or different UPDRS score parts among PD patients. These results are consistent with those revealed by MalecLitwinowicz and colleagues [24].

Our obtained results of the association between BRD subtype, increased disease duration, and increased salivary A-synolig level can be explained by the fact that Asynolig, that precedes the fibrillar aggregates found in LB, are the criminals for neuronal degeneration in PD [26, 27]. It was suggested that $L B$ themselves may be innocent in the PD pathogenesis [28]. Previous postmortem studies showed that LB were found in neurologically normal individuals over the age of 60 at high rate (approximately $10 \%)[29,30]$. Other studies revealed that the LB load detected in patients correlated poorly with the severity of symptoms [31, 32]. Finally, PD patients carrying familial mutations in the parkin gene, and some of those with the LRRK2 G2019S mutation, show neuronal degeneration in the absence of LB formation [33, 34]. Considering the results of previous studies together with the direct evidence demonstrating the toxicity of A-synolig, it was suggested that LB formation is a protective mechanism by acting as a sink for toxic oligomers, sequestering them away from the cellular machinery [35].

Interestingly, a recent study exhibited the role of VPS35 gene activity on A-syn degradation pathways that control the balance between the clearance and accumulation of A-syn. Thus, increasing VPS35 levels would be a future therapeutic tool in PD [36].

\section{Limitations of the study}

The saliva contains proteolytic and glycolytic enzymes that could influence the A-syn concentrations through proteolytic digestion. Despite specific precautions taken in the laboratory part of the study to prevent this enzymatic digestion, some residual enzymatic activity may be still present. Consequently, this will limit the ELISA results. A prospective longitudinal study conducted on a larger number of patients with long-term follow-up to investigate the relation between A-syn levels and disease severity and progression would provide more informative data. In addition, the patients included in the study were receiving different types of medications and their effect was not studied.

\section{Conclusion}

Salivary A-syn total and A-synolig might be considered potential biomarkers for PD diagnosis. Early diagnosis and intervention might help in preserving the neuronal functions, reducing the symptoms, slowing the disease progression, and improving the patients' quality of life.

Table 4 Comparisons of A-syn levels in the clinical phenotypes

\begin{tabular}{|c|c|c|c|c|c|c|}
\hline & \multicolumn{6}{|l|}{ A-syn } \\
\hline & \multicolumn{2}{|l|}{ A-synolig } & \multicolumn{2}{|l|}{ A-syn total } & \multicolumn{2}{|c|}{ A-synolig/A-syn total ratio } \\
\hline & Mean \pm SD & $p$ value & Mean \pm SD & $p$ value & Mean \pm SD & $p$ value \\
\hline$\overline{\mathrm{TD}}$ & $45.3 \pm 11.6$ & $0.03^{*}$ & $158.39 \pm 67.2$ & 0.9 & $0.35 \pm 0.2$ & 0.8 \\
\hline BRD & $57.7 \pm 6.5$ & & $161.4 \pm 36.4$ & & $0.37 \pm 0.09$ & \\
\hline
\end{tabular}

A-syn, alpha-synuclein; $A$-synolig, alpha-synuclein oligomer; $S D$, standard deviation; $T D$, tremor dominant; $B R D$, bradykinesia-rigidity dominant *Significant 


\section{Abbreviations}

A-syn: Alpha-synuclein; A-synmon: A-syn monomeric form; A-synolig: A-syn oligomer; BRD: Bradykinesia-rigidity dominant; CNS: Central nervous system; CSF: Cerebrospinal fluid; ELISA: Enzyme-linked immunosorbent assay; HRP: Horse radish peroxidase; HYS: Hoehn and Yahr scale; LB: Lewy bodies; LEDD: L-Dopa equivalent daily dose; NPP: Negative predictive value; PD: Parkinson's disease; PPV: Positive predictive value; ROC: Receiver operating characteristic; SD: Standard deviation; SPSS: Statistical Package for the Social Sciences; TD: Tremor-dominant; UPDRS: Unified Parkinson's Disease Rating Scale

\section{Acknowledgements}

We acknowledge Dr. Asmaa Younes, lecturer of Community, Faculty of Medicine, Fayoum University, for her effort in applying the statistical analysis of the study.

\section{Authors' contributions}

MA recruited the cases and collected all the needed data. SM, SS, and HS revised the clinical data obtained and the results. MM was responsible for the laboratory part of the study. Finally, MA and SM wrote the manuscript that was revised by the other authors to be ready for publication. SM is the corresponding author who is responsible for the publication.

\section{Funding}

No funding was obtained from any institution for our study.

\section{Availability of data and materials}

Data can be available for publication only by special approval from the Fayoum University.

\section{Ethics approval and consent to participate}

The study was approved by the Faculty of Medicine Research Ethical Committee of Fayoum University in its 26th session on 19 June 2016, number D93. A written consent was taken from all the participants either cases or controls after being informed about the objectives of the study, the examination, and the investigations. The confidentiality of their information was respected and their right not to participate in the study was ensured.

\section{Consent for publication}

Not applicable

\section{Competing interests}

None of the authors have any competing interests (financial or non-financial)

Received: 8 December 2019 Accepted: 31 January 2020

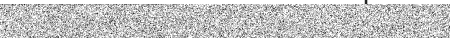

\section{References}

1. Oikonomou E, Paparrigopoulos T. Neuropsychiatric manifestations in Parkinson's disease. Psychiatriki. 2015;26(2):116-30.

2. Atik A, Stewart T, Zhang J. Alpha-synuclein as a biomarker for Parkinson's disease. Brain Pathol. 2016;26(3):410-8.

3. Fauvet B, Mbefo K, Fares B, Desobry C, Michael S, Ardah T, et al. a-Synuclein in central nervous system and from erythrocytes, mammalian cells, and Escherichia coli exists predominantly as disordered monomer. J Biol Chem. 2012;287:15345-64.

4. Vekrellis K, Xilouri M, Emmanouilidou E, Rideout J, Stefanis L. Pathological roles of a-synuclein in neurological disorders. Lancet Neurol. 2011;10:1015-25.

5. Roberts L, Brown R. Seeking a mechanism for the toxicity of oligomeric asynuclein. Biomolecules. 2015;5(2):282-305.

6. Beach $G$, Adler $H$, Sue I, Vedders L, Lue L, White lii $L$, et al. Multi-organ distribution of phosphorylated a-synuclein histopathology in subjects with Lewy body disorders. Acta Neuropathol. 2010;119(6):689-702.

7. Cersosimo M, Perandones C, Micheli F, Raina G, Beron A, Nasswetter G, et al. Alpha-synuclein immunoreactivity in minor salivary gland biopsies of Parkinson's disease patients. Mov Disord. 2011:26(1):188-90.

8. Devic I, Hwang H, Edgar S, Izutsu K, Presland R, Pan C, et al. Salivary asynuclein and DJ-1: potential biomarkers for Parkinson's disease. Brain. 2011; 134:e178.
9. Vivacqua G, Latorre A, Suppa A, Nardi M, Pietracupa S, Mancinelli R, et al. Abnormal salivary total and oligomeric alpha-synuclein in Parkinson's disease. PLoS One. 2016;11(3):e0151156.

10. Bougea A, Koros C, Stefanis L. Salivary alpha-synuclein as a biomarker for Parkinson's disease: a systematic review. J Neural Transm (Vienna). 2019; 126(11):1373-82

11. Hughes A, Daniel S, Blankson S, Lees A. A clinicopathologic study of 100 cases of Parkinson's disease. Arch Neurol. 1993:50(2):140-8.

12. Goetz CG, Fahn S, Martinez-Martin P, Poewe W, Sampaio C, Stebbins GT. Movement Disorder Society-sponsored revision of the Unified Parkinson's Disease Rating Scale (MDS-UPDRS): process, format, and clinimetric testing plan. Mov Disord. 2007;22(1):41-7.

13. Goetz CG, Poewe W, Rascol O, Sampaio C, Stebbins GT, Counsell C, et al. Movement Disorder Society Task Force report on the Hoehn and Yahr staging scale: status and recommendations. Mov Disord. 2004;19(9):1020-8.

14. Jankovic J, McDermott M, Carter J, Gauthier S, Goetz C, Golbe L, et al. Variable expression of Parkinson's disease: a base-line analysis of the DATATOP cohort. The Parkinson Study Group. Neurology. 1990;40(10):1529-34.

15. Tomlinson CL, Stowe R, Patel S, Rick C, Gray R, Clarke CE. Systematic review of levodopa dose equivalency reporting in Parkinson's disease. Mov Disord. 2010:25(15):2649-53.

16. El-Agnaf M, Salem A, Paleologou E, Curran D, Gibson J, Court A, et al. Detection of oligomeric forms of alpha-synuclein protein in human plasma as a potential biomarker for Parkinson's disease. FASEB J. 2006;20(3):419-25.

17. Tokuda T, Qureshi M, Ardah M, Varghese S, Shehab S, Kasai T, et al. Detection of elevated levels of alpha-synuclein oligomers in CSF from patients with Parkinson disease. Neurology. 2010;75:1766-72.

18. Park M, Cheon S, Bae H, Kim S, Kim J. Elevated levels of a-synuclein oligomer in the cerebrospinal fluid of drug-naïve patients with Parkinson's disease. J Clin Neurol. 2011;7(4):215-22.

19. Bengoa-Vergniory N, Roberts R, Wade-Martins R, Alegre-Abarrategui J. Alphasynuclein oligomers: a new hope. Acta Neuropathol. 2017;134(6):819-38.

20. Fairfoul G, McGuire L, Pal S, Ironside J, Neumann J, Christie S, et al. Alphasynuclein RT-QulC in the CSF of patients with alpha-synucleinopathies. Ann Clin Transl Neurol. 2016;3:812-7.

21. Farah $R$, Haraty H, Salame Z, Fares Y, Ojcius DM, Said SN. Salivary biomarkers for the diagnosis and monitoring of neurological diseases. Biomed J. 2018;41(2):63-87.

22. Thenganatt MA, Jankovic J. Parkinson disease subtypes. JAMA Neurol. 2014; 71(4):499-504

23. Paulus $W$, Jellinger $K$. The neuropathologic basis of different clinical subgroups of Parkinson'sdisease. J Neuropathol Exp Neurol. 1991;50(6):743-55.

24. Malec-Litwinowicz M, Plewka A, Plewka D, Bogunia E, Morek M, Szczudlik A, et al. The relation between plasma a-synuclein level and clinical symptoms or signs of Parkinson's disease. Neurol Neurochir Pol. 2018;52(2):243-51.

25. Shi M, Zabetian P, Hancock M, Ginghina C, Hong Z, Yearout D, et al. Significance and confounders of peripheral DJ-1 and alpha-synuclein in Parkinson's disease. Neurosci Lett. 2010:480:78-82.

26. Winner B, Jappelli R, Maji S, Desplats A, Boyer L, Aigner S, et al. In vivo demonstration that alpha-synuclein oligomers are toxic. Proc Natl Acad Sci USA. 2011;108(10):4194-9.

27. Helwig M, Klinkenberg M, Rusconi R, Musgrove R, Majbour N, El-Agnaf O, et al. Brain propagation of transduced a-synuclein involves non-fibrillar protein species and is enhanced in a-synuclein null mice. Brain. 2016:139(3):856-70.

28. Caughey B, Lansbury P. Protofibrils, pores, fibrils, and neurodegeneration: separating the responsible protein aggregates from the innocent bystanders. Annu Rev Neurosci. 2003;26:267-98.

29. Parkkinen L, Pirttilä T, Tervahauta M, Alafuzoff I. Widespread and abundant alpha-synuclein pathology in a neurologically unimpaired subject. Neuropathology. 2005;25(4):304-14

30. Frigerio R, Fujishiro H, Ahn T, Josephs K, Maraganore D, DelleDonne A, et al. Incidental Lewy body disease: do some cases represent a preclinical stage of dementia with Lewy bodies? Neurobiol Aging. 2011;32(5):857-63.

31. Colosimo C, Hughes A, Kilford L, Lees A. Lewy body cortical involvement may not always predict dementia in Parkinson's disease. J Neurol Neurosurg Psychiatry. 2003;74:852-6.

32. Parkkinen L, Pirttila T, Alafuzoff I. Applicability of current staging/ categorization of alpha-synuclein pathology and their clinical relevance. Acta Neuropathol. 2008;115(4):399-407.

33. Gaig C, Martí M, Ezquerra M, Cardozo A, Rey M, Tolosa E. G2019S LRRK2 mutation causing Parkinson's disease without Lewy bodies. J Neurol Neurosurg Psychiatry. 2007;78(6):626-8. 
34. Cookson M, Hardy J, Lewis P. Genetic neuropathology of Parkinson's disease. Int J Clin Exp Pathol. 2008;1(3):217-31.

35. Soto C, Estrada L. Protein misfolding and neurodegeneration. Arch Neurol. 2008:65(2):184-9.

36. Eleuteri S, Albanese A. VPS35-based approach: a potential innovative treatment in Parkinson's disease. Front Neurol. 2019;10:1272.

\section{Publisher's Note}

Springer Nature remains neutral with regard to jurisdictional claims in published maps and institutional affiliations.

Submit your manuscript to a SpringerOpen ${ }^{\mathcal{O}}$ journal and benefit from:

- Convenient online submission

- Rigorous peer review

- Open access: articles freely available online

High visibility within the field

- Retaining the copyright to your article

Submit your next manuscript at $\boldsymbol{\sim}$ springeropen.com 